\title{
Extradural Transjugular Transsigmoid Approach with High Cervical Exposure for Glomus Jugulare Tumor
}

\author{
Ken Matsushima ${ }^{1}$ Michihiro Kohno ${ }^{1}$ Takao Hashimoto ${ }^{1}$ Hitoshi Izawa ${ }^{1}$ Yujiro Tanaka ${ }^{1}$ \\ Taro Inagaki \\ 1 Department of Neurosurgery, Tokyo Medical University, Tokyo, Japan \\ 2 Department of Otorhinolaryngology, Head and Neck Surgery, Tokyo \\ Medical University, Tokyo, Japan \\ Address for correspondence Michihiro Kohno, MD, PhD, Department \\ of Neurosurgery, Tokyo Medical University, \#6-7-1 Nishishinjuku, \\ Shinjuku-ku, Tokyo 160-0023, Japan \\ (e-mail: mkouno-nsu@umin.ac.jp).
}

J Neurol Surg B 2019;80(suppl S4):S382-S384.

\begin{abstract}
Surgical removal of glomus jugulare tumors is challenging owing to the complex anatomy of the temporal bone and craniocervical region, aggressive tumor invasion into the nearby structures, and their hypervascularity. However, recent advances in skull base techniques, intraoperative neuromonitoring, and radiological interventions have enabled their relatively safe resection, while giving priority to functional preservation. This video demonstrates a case of a glomus jugulare tumor treated by the extradural transjugular transsigmoid approach with high-cervical exposure and tympamoplasty, after preoperative embolization. A 47-year-old woman presented with progressive hearing disturbance, pulsatile tinnitus, and hemifacial spasm. Neuroimaging displayed a hypervascular tumor occupying the temporal bone, extending to the cervical region through the jugular foramen, and to the external auditory canal. Preoperative feeder occlusion was successfully performed without any additional symptoms, while carefully evaluating the provocative test. Near-total resection of the tumor was achieved through the transjugular transsigmoid approach with high-cervical

\section{Keywords}

- head and neck

- infralabyrinthine approach

- infratemporal fossa

- jugulotympanic paraganglioma

- postauricular transtemporal exposure under detailed neuromonitoring, including continuous facial nerve monitoring and auditory brainstem response. In this patient, in whom the tumor did not invade intradurally and the sigmoid sinus was already occluded preoperatively, the sinus was managed only by coagulation, to avoid unnecessary dural opening and the risk of cerebrospinal fluid leakage. Anterior facial nerve rerouting was not required since the tumor removal was accomplished through the corridor above and below the fallopian bridge. The patient had no new neurological deficits, and her pulsatile tinnitus and hemifacial spasm disappeared after the surgery. Her hearing disturbance improved postoperatively.

The link to the video can be found at: https://youtu.be/gqf3dxHlv_0.
\end{abstract}

received

March 1, 2019

accepted

July 9, 2019

published online

October 3, 2019 www.thieme.com/skullbasevideos

www.thieme.com/jnlsbvideos
License terms

Stuttgart · New York 10.1055/s-0039-1695057.

ISSN 2193-6331. 

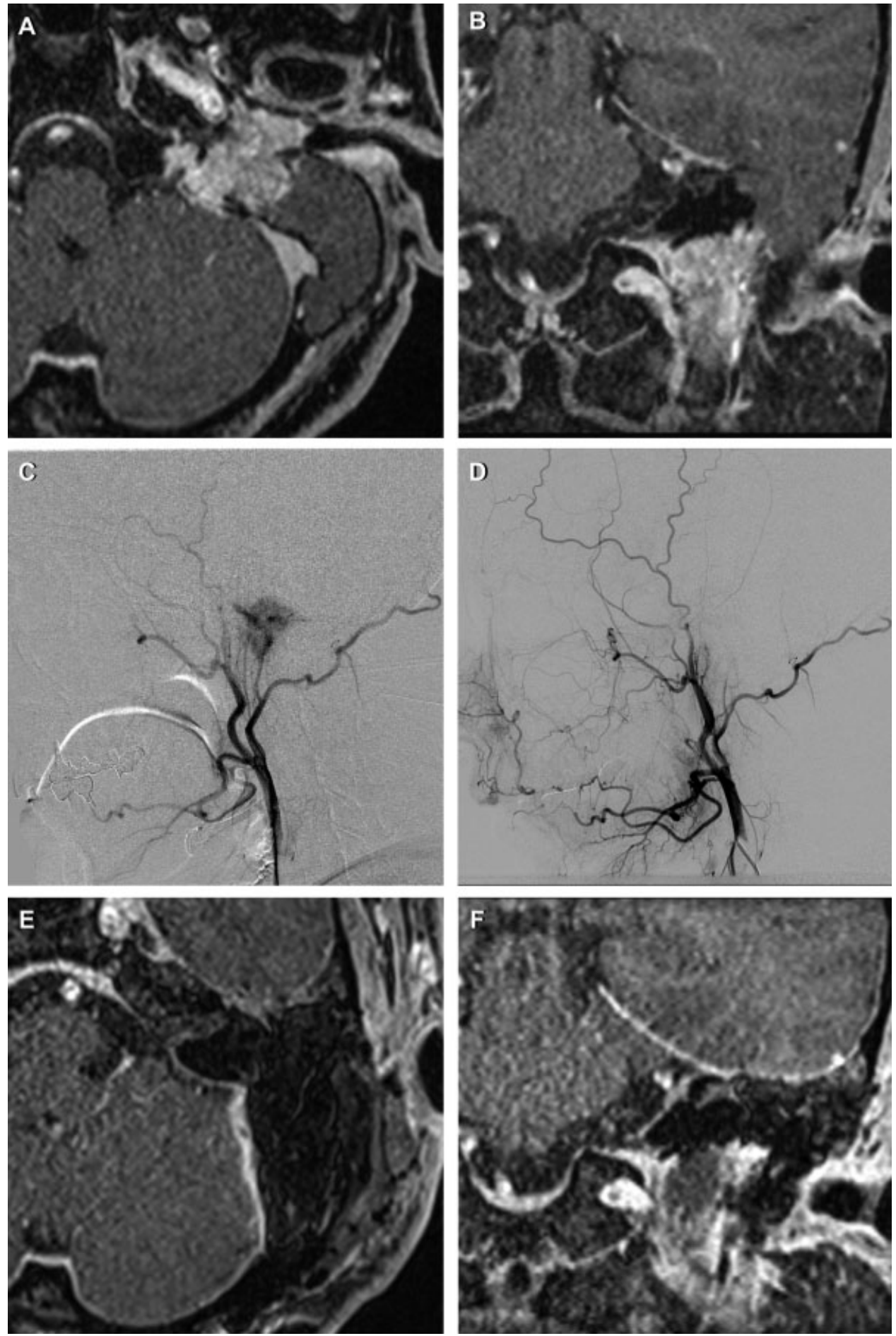

Fig. 1 Preoperative post-gadolinium T1-weighted MRIs show a left glomus jugulare tumor extending extracranially (A and B). The tumor was highly vascularized by the stylomastoid, ascending pharyngeal, and posterior auricular arteries (C), and preoperative embolization significantly decreased the tumor staining (D). Postoperative MRIs confirmed successful resection through the transjugular transsigomid approach (E and F). 

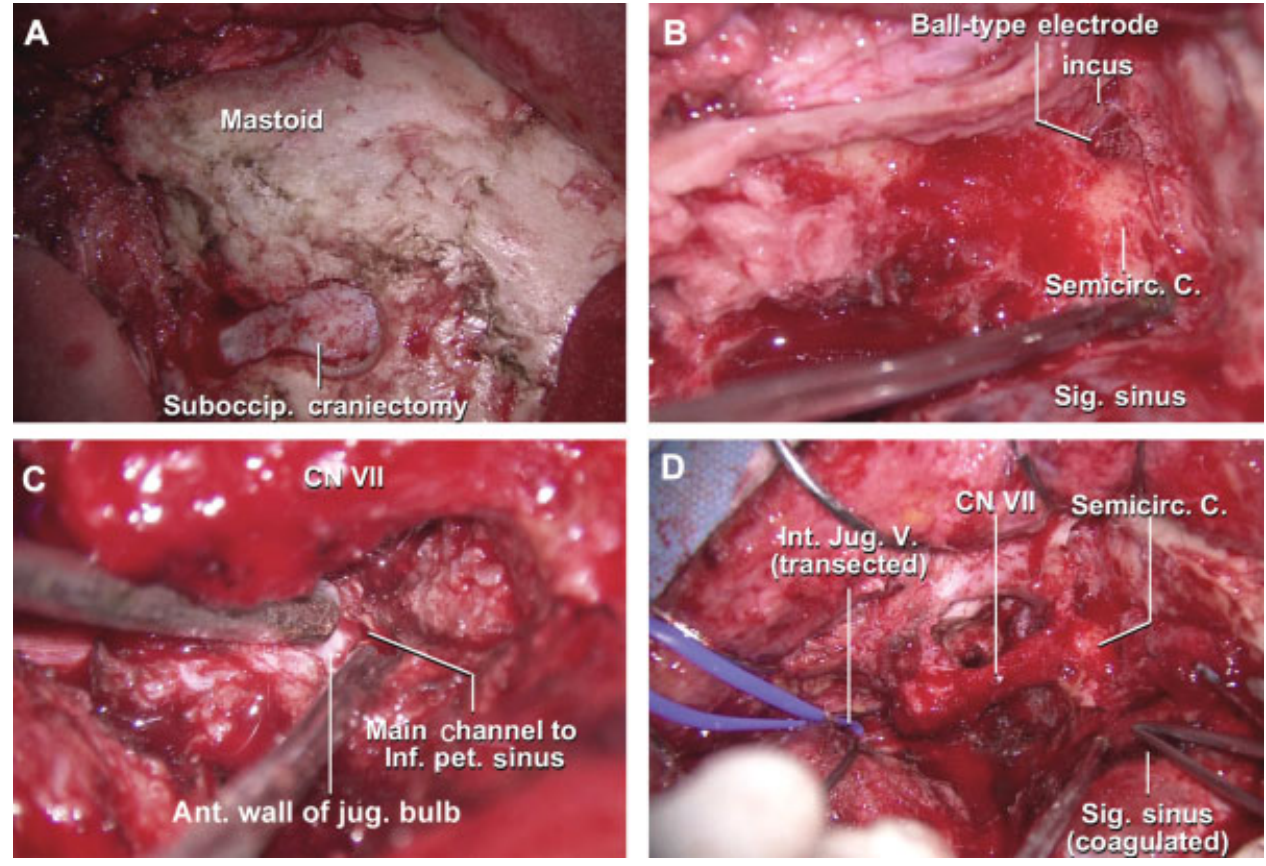

Fig. 2 (A) The left mastoid bone was exposed and small suboccipital craniectomy was performed. (B) To continuously assess the neural condition in real-time without disturbing the microsurgical procedure, the evoked facial electromyogram was monitored with electrical stimulation at a frequency of $1 \mathrm{~Hz}$ throughout the procedure, with a ball-type stimulating electrod placed in the aditus. (C) The intrajugular tumor was dissected, while preserving the anterior wall of the jugular bulb. Venous flow from the inferior petrosal sinus was encountered, and the hemorrhage was promptly controlled using fibrin glue-soaked hemostatic material. (D) The intrapetrous tumor was resected through the corridor above and below the fallopian bridge, while preserving the facial nerve and semicircular canals. The intrajugular and extracranial tumors were also resected after ligation of the internal jugular vein. In this patient, in which the tumor did not invade intradurally and the sigmoid sinus was already occluded preoperatively, the sinus was managed only by coagulation, to avoid unnecessary dural opening and the risk of cerebrospinal fluid leakage. Ant., anterior; C., canal; CN, cranial nerve; Inf., inferior; Int., internal; Jug., jugular; Pet., petrosal; Semicirc., semicircular; Sig., sigmoid; Suboccip., suboccipital; V., vein.

\section{Conflict of Interest}

The authors have no conflicts of interest associated with this study.

\section{Acknowledgments}

We thank Ms. Miki Hioki and Mr. Yoshifumi Kawaguchi for their support as medical technologists.

\section{References}

1 Liu JK, Sameshima T, Gottfried ON, Couldwell WT, Fukushima T. The combined transmastoid retro- and infralabyrinthine transjugular transcondylar transtubercular high cervical approach for resection of glomus jugulare tumors. Neurosurgery 2006;59(01, Suppl 1):ONS115-ONS125, discussion ONS115-ONS125

2 Mann WJ, Amedee RG, Gilsbach J, Perneczky A, Wolfensberger M. Transsigmoid approach for tumors of the jugular foramen. Skull Base Surg 1991;1(03):137-141 